\title{
Synthesis and Self-Assembly Behavior of Poly(fluorenylstyrene)-block- poly(2-vinylpyridine) Block Copolymers and Their Blends with Single Wall Carbon Nanotubes (SWCNTs)
}

\author{
Chaoxu Li, ${ }^{\dagger}$ Jung-Ching Hsu, ${ }^{\ddagger}$ Kenji Sugiyama,,${ }^{\S}$ Akira Hirao, ${ }^{\S}$ Wen-Chang Chen, ${ }^{*} \star$ and \\ Raffaele Mezzenga $*, \dagger, \perp$ \\ †Department of Physics and Fribourg Center for Nanomaterials, University of Fribourg, Chemin du Musée 3, \\ 1700 Fribourg, Switzerland, 'Department of Chemical Engineering, National Taiwan University, No. 1, Sec. 4, \\ Roosevelt Road, Taipei 10617, Taiwan, ${ }^{\S}$ Department of Organic and Polymeric Materials, Graduate School \\ of Science and Engineering, Tokyo Institute of Technology, H-127, 2-12-1 Ohokayama, Meguro-ku, Tokyo \\ 152-8552, Japan, and ${ }^{\perp}$ Nestlé Research Center, Vers-chez-les-blanc, 1000 Lausanne 26, Switzerland
}

\begin{abstract}
We have synthesized a series of poly[4-(9,9-dihexylfluoren-2-yl)styrene]-block-poly(2-vinylpyridine) (P(St-F1)-b-P2VP) and poly[4-di(9,9-dihexylfluoren-2-yl)styrene]-block-poly(2-vinylpyridine) $\left(\mathrm{P}\left(\mathrm{St}-\mathrm{F} 1_{2}\right)-b-\mathrm{P} 2 \mathrm{VP}\right)$ comblike block copolymers and investigated their self-assembly behavior. The block composition, molecular weight, and the length of the fluorene side chain were varied to control the final morphologies. Although it was found that side chains composed by up to two fluorene units were not sufficient to induce any liquid crystalline structure, the phase diagrams of the resulting block copolymers greatly depended on fluorene content. Depending on the number of pendant fluorene units, the block volume ratio, the solvent used to cast the samples, and thermal annealing history, spherical, hexagonally packed cylinders, lamellar, gyroidal, and hexagonally perforated lamellae could be found. The resulting phase diagram, compared to the reference PS- $b$-P2VP system, was interpreted in terms of the conformational asymmetry of block copolymers arising from the grafting of fluorene units of variable lengths onto the backbone of the PS block. The capability of $\mathrm{P}\left(\mathrm{St}-\mathrm{F} 1_{n}\right)-b$-P2VP to host $\mathrm{COOH}$-modified single wall carbon nanotubes (SWCNTs) was also investigated. It was found that hydrogen bonding between $\mathrm{COOH}$ and P2VP favors miscibility of SWCNT within P2VP domains, and the blending of these two components was reflected both on the final morphologies of the block copolymers and on the electron conductivity of the SWCNTblock copolymer blends.
\end{abstract}

\section{Introduction}

Diblock copolymers are increasingly receiving renewed attention in the field of nanodevice applications due to their ability of ordering into various periodic structures. ${ }^{1}$ To achieve the desired functions, the morphology of block copolymers usually requires to be precisely controlled. In principle, this can be realized to a good extent by tuning two main quantities: the block composition $(f)$ and degree of segregation $(\chi N){ }^{2}$ However, in order to obtain suitable properties in practical real applications, rigid or semiflexible blocks, segments or mesogens need to be incorporated into the molecular design, most commonly as side chains or main chain extensions, leading to richer and more complex morphologies. $^{3}$

One common pathway to increase morphological complexity in polymers and block copolymers is the use of comb-type architectures in which pencil flexible or rigid moieties emanate out of a main soft backbone. The resulting polymers usually possess high chain rigidity ${ }^{4}$ and liquid crystalline nature, ${ }^{5}$ depending on the length and the chemical structure of side chains. By varying the short alkyl side chains of weakly interacting polystyrene(PS)-block-poly(alkyl methacrylate), Mayes et al. found that their phase behavior could be greatly altered. ${ }^{6}$ By supramolecularly and selectively attaching an alkyl side chain of varying length to PS-block-poly(4-vinylpyridine), Ikkala and

\footnotetext{
*To whom correspondence should be addressed.
}

co-workers observed a series of structure-within-structures with liquid crystalline features at the short length scale. ${ }^{7}$ The engineering of comblike supramolecular liquid crystalline polymers was further expanded by the group of one of the present authors to cationic dendronized polymers, dendrons, and dendrimers, leading to new types of columnar liquid crystalline phases ${ }^{8}$ and new families of structure-within-structures hierarchical morphologies. ${ }^{9}$

In conjugated polymers, such as polyfluorenes, polythiophenes, or polyphenylenes, the use of side chains is usually employed to provide enhanced solubility and, thus, their improved processability into light-emitting diodes and photovoltaic devices. In the solid state, hierarchical self-assembly and side chain crystallization are possible also in these systems. ${ }^{10}$

In this study, we describe the synthesis of a series of poly[4-(9,9dihexylfluoren-2-yl)styrene]-block-poly(2-vinylpyridine) (P(St-F1)$b$-P2VP) and poly[4-di(9,9-dihexylfluoren-2-yl)styrene]-blockpoly(2-vinylpyridine) $\left(\mathrm{P}\left(\mathrm{St}-\mathrm{F} 1_{2}\right)-b\right.$ - $\left.\mathrm{P} 2 \mathrm{VP}\right)$. The block composition, molecular weight, and the length of the fluorene side chain were varied in order to control the final morphologies. Although it was found that side chains composed by up to two fluorene units are not sufficient to induce any liquid crystalline structure, the phase diagrams of block copolymers results greatly changed.

Furthermore, we study the effect of blending single walled carbon nanotubes (SWCNTs) into the P2VP domains of the microphase-separated block copolymers as a possible way to alter 
the electronic properties of blends. A few examples of dispersing carbon nanotubes (CNTs) into block copolymers have been already reported. ${ }^{11}$ In general, however, due to the strong van der Waals and $\pi-\pi$ interactions among CNTs, their dispersion into polymer matrices is usually very difficult. Kong et al. proposed to graft block copolymers on the surface of CNTs by atom transfer radical polymerization to prepare nanocomposites. $^{12}$ Zou et al. found a general strategy of dispersing CNTs by using conjugated block copolymers. ${ }^{11 \mathrm{~b}}$ However, these reports do not discuss the interplay of the CNTs orientation and the morphologies of the final block copolymer-CNTs blends. Here we show that by using $\mathrm{COOH}$-modified single wall carbon nanotubes, the hydrogen bonding between the carboxylic and vinylpyridine groups is sufficient to disperse SWCNTs within the P2VP domains, although in low amount ( $\approx 2 \mathrm{wt} \%)$. Also, the impact of the presence of SWCNTs in the final block copolymer blends is discussed by combining transmission electron microscopy images and electron semiconductivity measurements.

\section{Experimental Section}

2.1. Materials. Reagents were purchased from Aldrich or Acros and used as received, unless otherwise stated. Monomers of St-Fl and $\mathrm{St}-\mathrm{Fl}_{2}$ were synthesized according to the literature. $^{13,14}$ 1,1-Diphenylethylene (DPE) and 2-vinylpyridine were purified according to the usual procedure and were finally distilled twice over $\mathrm{CaH}_{2}$ under a nitrogen atmosphere. Tetrahydrofuran (THF) was refluxed over sodium wire, distilled over $\mathrm{LiAlH}_{4}$ under nitrogen, and then distilled from its sodium naphthalenide solution under high vacuum conditions $\left(10^{-6}\right.$ Torr). Heptane was washed with concentrated $\mathrm{H}_{2} \mathrm{SO}_{4}$, water, and aqueous $\mathrm{NaHCO}_{3}$, dried over $\mathrm{P}_{2} \mathrm{O}_{5}$, and finally distilled in the presence of 1,1-diphenylhexyllithium. sec-Butyllithium (secBuLi, 1.2 $\mathrm{M}$ in heptane, Aldrich, Japan) was diluted with heptane and determined in concentration by the colorimetric titration from colorless end point of the red solution obtained after the addition of 1,1-diphenylethylene with standardized octanol in THF solution under high-vacuum conditions.

2.2. General Procedure for the Synthesis of $P\left(S t-F l_{n}\right)-b-P 2 V P$ $(n=1,2)$ Block Copolymers. The $\mathrm{P}\left(\mathrm{St}_{-}-\mathrm{Fl}_{n}\right)-b$-P2VP $(n=1,2)$ diblock copolymer was prepared by the sequential addition of $\mathrm{St}_{-} \mathrm{Fl}_{n}(n=1,2), \mathrm{DPE}$, and 2-vinylpyridine. Afterward, the reaction was quenched with degassed methanol. The first stage polymerization was carried out at $-78^{\circ} \mathrm{C}$ by mixing $\mathrm{St}-\mathrm{Fl} l_{n}(n=$ $1,2)$ in THF solution with sec-BuLi in heptane solution. The reaction mixture was allowed to stand for $3 \mathrm{~h}$. Then, DPE in THF solution was added to the reaction reactor under the same condition for an additional $30 \mathrm{~min}$. Finally, 2-vinylpyridine in THF solution was added to the resulting living poly $\left(\mathrm{St}-\mathrm{Fl}_{n}\right)$ solution obtained at the first stage polymerization. After $30 \mathrm{~min}$, the reaction mixture was quenched with degassed methanol, and the polymer was concentrated using a rotavap drying system. The polymer was purified by washing twice with $n$-hexane or ethyl ether and finally freeze-dried from its benzene solution. The specific amount of initiator and monomers added in the reaction, molecular weight, and composition measured by SEC and ${ }^{1} \mathrm{H}$ NMR are listed in Table 1.

Poly(St-Fl)-block-(2-vinylpyridine): $300 \mathrm{MHz}{ }^{1} \mathrm{H} \quad \mathrm{NMR}$ $\left(\mathrm{CDCl}_{3}, \mathrm{ppm}\right)$ : 8.4-8.0 (pyridine ring aromatic protons), 7.76.2 (broad, aromatic), 2.8-0.5 (broad, backbone and $\mathrm{C}_{6} \mathrm{H}_{13}$ ).

Poly(St-Fl 2 -block-(2-vinylpyridine): $300 \mathrm{MHz}{ }^{1} \mathrm{H}$ NMR $\left(\mathrm{CDCl}_{3}, \mathrm{ppm}\right)$ : 8.5-8.0 (pyridine ring aromatic protons), $7.8-$ 6.2 (broad, aromatic protons), 2.5-0.6 (broad, backbone and $\mathrm{C}_{6} \mathrm{H}_{13}$ ).

2.3. Characterization of Chemical Structure of Block Copolymers. Size exclusion chromatography (SEC) was performed on an Asahi Techneion AT-2002 equipped with a Viscotek TDA model 302 triple detector array using THF as a carrier solvent at a flow rate of $1.0 \mathrm{~mL} / \mathrm{min}$ at $40{ }^{\circ} \mathrm{C}$. To determine $M_{\mathrm{n}}$ and
$M_{\mathrm{w}} / M_{\mathrm{n}}$ values of the resulting polymers, a calibrated curve was made with polystyrene standard. ${ }^{1} \mathrm{H}$ NMR spectra were recorded on a Bruker DPX (300 MHz for $\left.{ }^{1} \mathrm{H}\right)$ in $\mathrm{CDCl}_{3}$.

2.4. Polymer-Carbon Nanotube Composite Preparation. Carboxylic single-wall carbon nanotubes (P3-SWCNT, containing 4-6 atom \% carboxylic acid) were obtained from Carbon Solutions, Inc., and were used without further purification. First, solutions of block copolymers in DMF were prepared (0.5 wt \%). P3-SWCNTs were dispersed in DMF (0.5 wt \%) using sonication for $1 \mathrm{~h}$. After a predetermined amount of P3SWCNT mixture was dropped into the block copolymer solution, the obtained mixture was further sonicated for $30 \mathrm{~min}$ and then centrifuged at $6000 \mathrm{rpm}$ for $1 \mathrm{~h}$ to remove the insoluble part of carbon nanotubes. Thereafter, the mixture was cast on a Teflon membrane at $60{ }^{\circ} \mathrm{C}$ (without further specification, the thickness of solution-cast samples in this study was higher than $100 \mu \mathrm{m})$. The casting sample was further dried under high vacuum (ca. $10^{-8} \mathrm{mbar}$ ) at $70^{\circ} \mathrm{C}$ for $24 \mathrm{~h}$.

2.5. Conductivity Measurements. The ac conductivity was measured via an electric bridge measurement with an oscilloscope (HP 54540C). Samples were cast as films (with the thickness of $80-100 \mu \mathrm{m}$ ) from block copolymer/SWCNT DMF mixtures onto glass substrates, which were partially sputtered by gold with a mask to yield four electrodes for four-probe conductivity measurements. After casting, the films were annealed at $170{ }^{\circ} \mathrm{C}$ for $24 \mathrm{~h}$ under high vacuum. An alternating voltage of $1-2 \mathrm{~V}$ was applied at a constant frequency of $50 \mathrm{~Hz}$. The conductivity $(\sigma)$ was obtained from $G=\sigma A / l$, where $G$ is the conductance, $A$ is the cross-sectional area, and $l$ is the gold electrode fixed distance.

2.6. Small-Angle X-ray Scattering (SAXS). X-ray scattering experiments were performed on an Anton Parr SAXSess system, operated in line-collimation geometry with $\lambda=0.154 \mathrm{~nm} \mathrm{X-rays}$ and a sample-to-detector (image plate) distance of $26 \mathrm{~cm}$, which gives an effective $q$-range $0.1-7 \mathrm{~nm}^{-1}$. Here $q$ is the scattering vector defined as $q=4 \pi \sin (\theta) / \lambda$, with $2 \theta$ the scattering angle.

2.7. Transmission Electron Microscopy (TEM). $50 \mathrm{~nm}$ thick sections for TEM were cut using a Leica Ultracut UCT ultramicrotome, collected onto 300-mesh copper grids and stained with iodine vapor for $4 \mathrm{~h}$ prior to analysis. Bright-field TEM images were taken using a CM100 Philips TEM operated at $80 \mathrm{kV}$. Images were acquired on a SIS Morada CCD camera.

\section{Results and Discussion}

3.1. Synthesis of Block Copolymers. Monomers of St-Fl and $\mathrm{St}-\mathrm{Fl}_{2}$ were synthesized according to the literature (see the Supporting Information). ${ }^{13,14}$ The synthetic scheme and detailed synthesis of the $\mathrm{St}-\mathrm{Fl}$ and $\mathrm{St}-\mathrm{Fl}_{2}$ are given in the Supporting Information. The block polymers of $\mathrm{P}(\mathrm{St}-\mathrm{Fl})$ block-P2VP and $\mathrm{P}\left(\mathrm{St}-\mathrm{Fl}_{2}\right)$-block-P2VP were synthesized by sequential living anionic polymerization as depicted in Scheme 1, similar to our recent study on the block copolymers of $\mathrm{P}(\mathrm{St}-\mathrm{Fl})$-block-polystyrene and $\mathrm{P}\left(\mathrm{St}-\mathrm{Fl}_{2}\right)$-blockpolystyrene. ${ }^{14}$ The amount of the initiator and the ratio of the monomers were carefully controlled to tune the molecular weight and block composition. Since the molecular weight on the first block of $\mathrm{P}(\mathrm{St}-\mathrm{Fl})$ or $\mathrm{P}\left(\mathrm{St}_{-} \mathrm{Fl}_{2}\right)$ could be accurately predicted from the feed ratios of the monomer to the initiator, ${ }^{14}$ it was not necessary to precipitate out from the polymerization, and we proceeded with polymerization of the second block. The chemical structures of the block copolymers synthesized were confirmed by ${ }^{1} \mathrm{H}$ NMR spectra. The representative ${ }^{1} \mathrm{H}$ NMR spectra are shown in Figure 1. The block composition was estimated by comparing the relative integration of the peaks from 7.76.2 and $8.5-8.0 \mathrm{ppm}$, assigned to the aromatic groups in the $\mathrm{P}\left(\mathrm{St}-\mathrm{Fl}_{n}\right)$ block and pyridine ring protons in the P2VP block, respectively. The number-average molecular weight $\left(M_{\mathrm{n}}\right)$ and polydispersity index (PDI) are determined by SEC 
Scheme 1. Synthetic Routes of the $\mathrm{P}\left(\mathrm{St}-\mathrm{Fl}_{n}\right)-b-\mathrm{P} 2 \mathrm{VP}(n=1,2)$ Diblock Copolymer

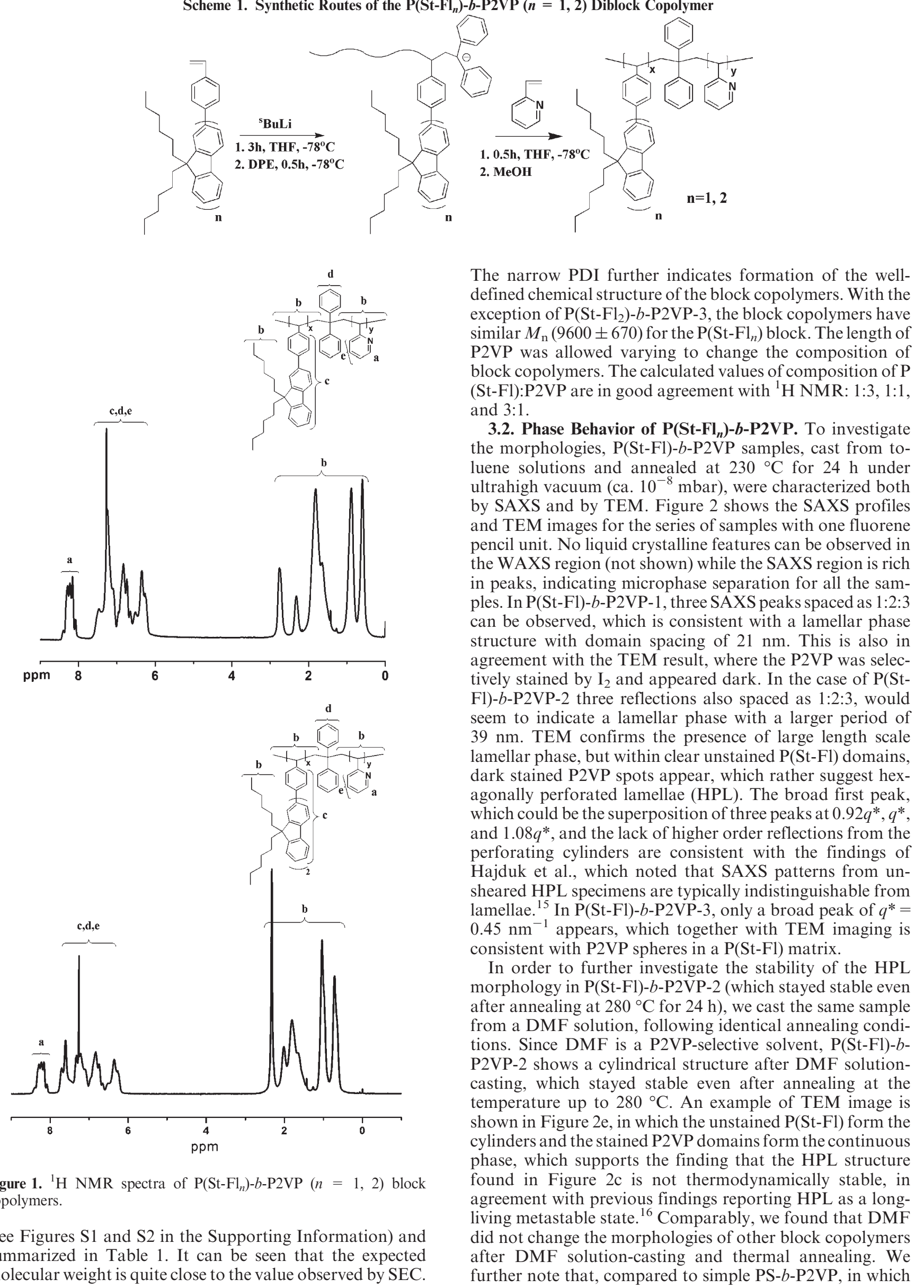


Table 1. Anionic Polymerization of $\mathrm{P}\left(\mathrm{St}_{-} \mathrm{Fl}\right)$ ) $-b-2 \mathrm{VP}(n=1,2)$ in THF at $-78{ }^{\circ} \mathrm{C}^{a}$

\begin{tabular}{|c|c|c|c|c|c|c|c|}
\hline \multirow[b]{2}{*}{ run } & \multirow[b]{2}{*}{ sec-BuLi (mmol) } & \multirow[b]{2}{*}{ monomers $\left(\mathrm{St}_{-} \mathrm{Fl}_{n} / \mathrm{DPE} / 2 \mathrm{VP}\right)(\mathrm{mmol})$} & \multicolumn{2}{|c|}{$M_{\mathrm{n}}(\mathrm{g} / \mathrm{mol})$} & \multirow[b]{2}{*}{$M_{\mathrm{w}} / M_{\mathrm{n}}$} & \multicolumn{2}{|c|}{ composition $\left(\mathrm{St}-\mathrm{Fl} l_{n} / 2-\mathrm{VP}\right)(\mathrm{w} / \mathrm{w})$} \\
\hline & & & calc & obs $^{b}$ & & calc & obs $^{c}$ \\
\hline $\mathrm{P}(\mathrm{St}-\mathrm{Fl})-b-\mathrm{P} 2 \mathrm{VP}-1$ & 0.084 & St-Fl, 1.87/0.19/7.92 & 19700 & 19700 & 1.04 & $0.49 / 0.51$ & $0.47 / 0.53$ \\
\hline $\mathrm{P}(\mathrm{St}-\mathrm{Fl})-b-\mathrm{P} 2 \mathrm{VP}-2$ & 0.039 & St-Fl, $0.874 / 0.159 / 10.9$ & 39200 & 39600 & 1.02 & $0.25 / 0.75$ & $0.26 / 0.74$ \\
\hline $\mathrm{P}(\mathrm{St}-\mathrm{Fl})-b-\mathrm{P} 2 \mathrm{VP}-3$ & 0.084 & St-Fl, $1.83 / 0.171 / 2.34$ & 12500 & 10400 & 1.08 & $0.76 / 0.24$ & $0.86 / 0.14$ \\
\hline $\mathrm{P}\left(\mathrm{St}-\mathrm{Fl}_{2}\right)-b-\mathrm{P} 2 \mathrm{VP}-1$ & 0.077 & St- $\mathrm{Fl}_{2}, 1.364 / 0.108 / 10.415$ & 27700 & 18000 & 1.05 & $0.49 / 0.51$ & $0.48 / 0.52$ \\
\hline $\mathrm{P}\left(\mathrm{St}-\mathrm{Fl}_{2}\right)-b-\mathrm{P} 2 \mathrm{VP}-2$ & 0.071 & $\mathrm{St}^{-\mathrm{Fl}_{2}}, 1.3590 .0748 / 3.96$ & 20500 & 13000 & 1.04 & $0.71 / 0.29$ & $0.73 / 0.27$ \\
\hline $\mathrm{P}\left(\mathrm{St}-\mathrm{Fl}_{2}\right)-b-\mathrm{P} 2 \mathrm{VP}-3$ & 0.773 & St-Fl $l_{2}, 0.485 / 0.133 / 10.4$ & 19000 & 17900 & 1.08 & $0.25 / 0.75$ & $0.27 / 0.73$ \\
\hline
\end{tabular}
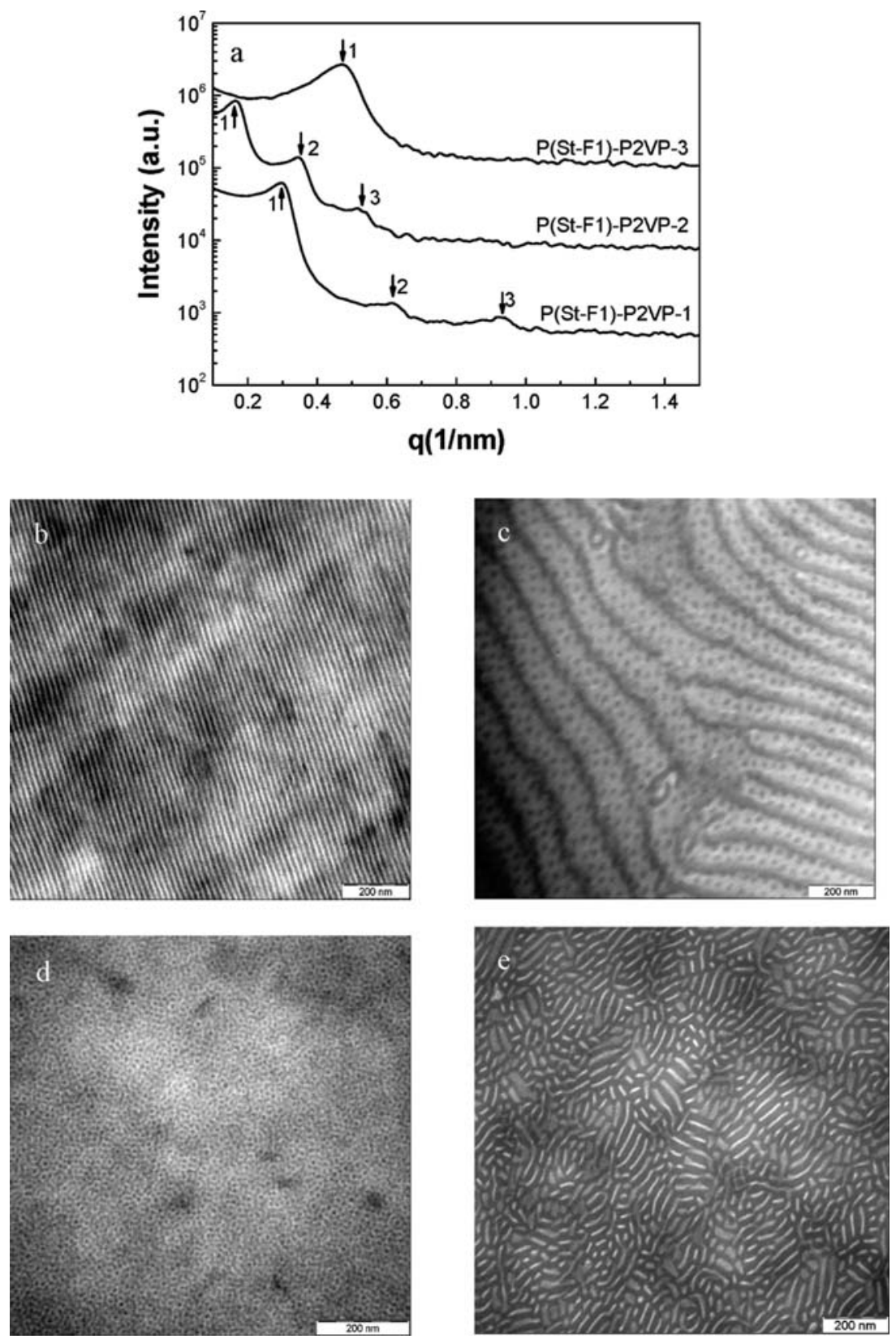

Figure 2. SAXS profiles (a) and TEM images ( $\mathrm{b}-\mathrm{d}$ ) of $\mathrm{P}(\mathrm{St}-\mathrm{Fl})-b$-P2VP casting from toluene and annealing at $230^{\circ} \mathrm{C}$ for $24 \mathrm{~h}$ : (b) $\mathrm{P}(\mathrm{St}-\mathrm{Fl})-b$ - $\mathrm{P} 2 \mathrm{VP}-1$, (c) P(St-Fl)- $b$-P2VP-2, (d) P(St-Fl)- $b$-P2VP-3. (e) TEM image of P(St-Fl)- $b$-P2VP-2 casting from DMF and annealing at $230^{\circ} \mathrm{C}$ for $24 \mathrm{~h}$.

HPL is found at the PS composition of $0.35-0.40,{ }^{17}$ the present system presents HPL phases at somewhat lower volume fraction, e.g., 0.28 of $\mathrm{P}(\mathrm{St}-\mathrm{F} 1)$ in $\mathrm{P}(\mathrm{St}-\mathrm{Fl})-b-\mathrm{P} 2 \mathrm{VP}-2$.

Moreover, it was found that three $\mathrm{P}(\mathrm{St}-\mathrm{Fl})-b$-P2VP block copolymers lay in the ordered state until $280{ }^{\circ} \mathrm{C}$, which suggests that their order-to-disorder transition temperatures $\left(T_{\text {ODT }} \mathrm{s}\right)$ are higher than $280{ }^{\circ} \mathrm{C}$. By using the temperature dependence of the interaction parameter $(\chi), \operatorname{Schulz}^{17}$ and Zha ${ }^{18}$ predicted successfully the $T_{\text {ODT }}$ of PS- $b$-P2VP; for a PS- $b$-P2VP with the composition $(0.5)$ and $M_{\mathrm{n}}$ (ca. 20000) similar to $\mathrm{P}(\mathrm{St}-\mathrm{Fl})-b-\mathrm{P} 2 \mathrm{VP}-1$, the $T_{\text {ODT }}$ was reported to be ca. $200{ }^{\circ} \mathrm{C}$. Clearly, the addition of the fluorene side chain units in the present case increases the degree of segregation $(N \chi)$ of the block copolymer, leading to higher $T_{\mathrm{ODT}}$, 

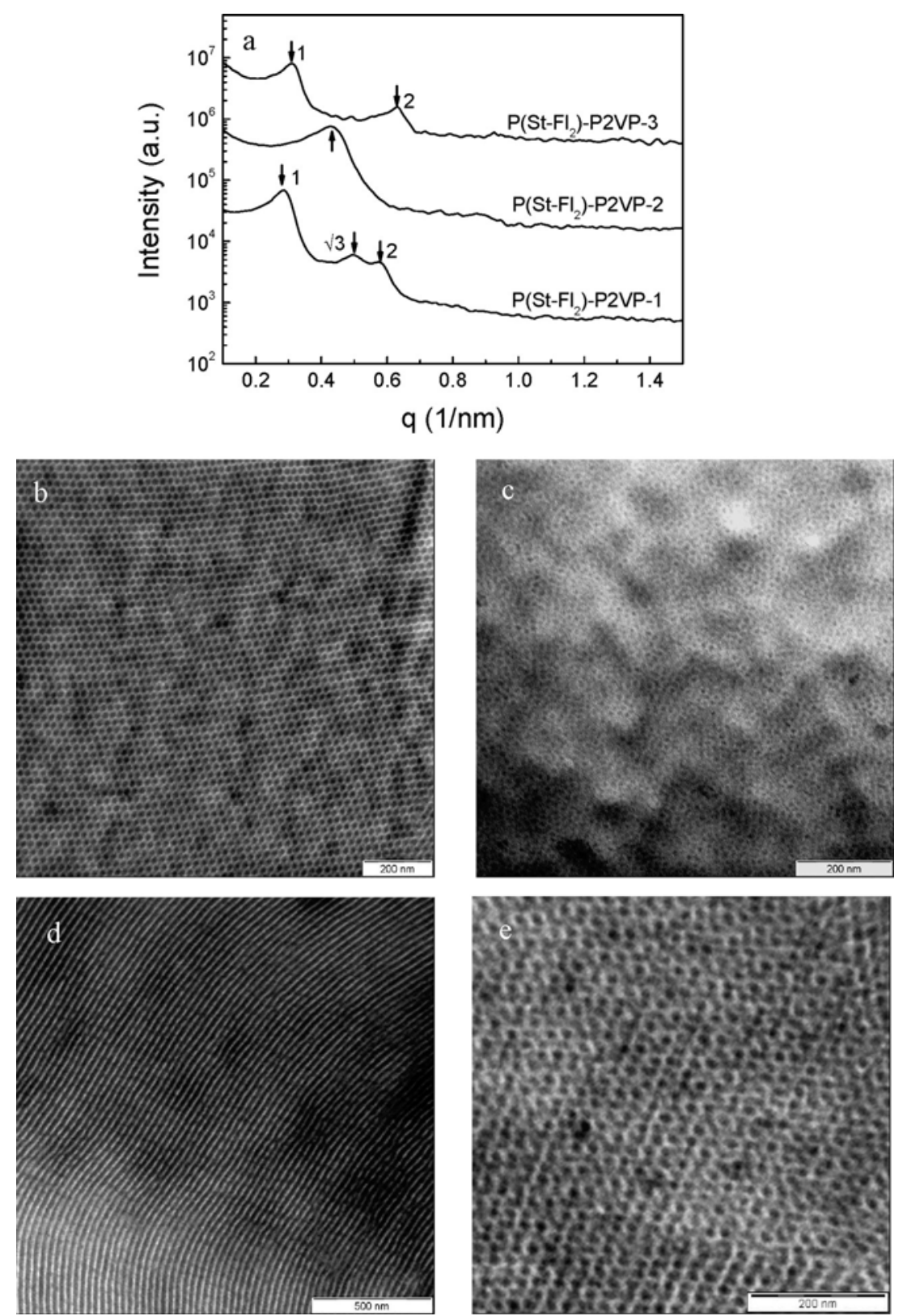

Figure 3. SAXS profiles (a) and TEM images $(\mathrm{b}-\mathrm{d})$ of $\mathrm{P}\left(\mathrm{St}_{-}-\mathrm{Fl}_{2}\right)-b-\mathrm{P} 2 \mathrm{VP}$ casting from toluene and annealing at $230{ }^{\circ} \mathrm{C}$ for $24 \mathrm{~h}$ : (b) $\left.\mathrm{P}(\mathrm{St}-\mathrm{Fl})_{2}\right)-b$ - $\mathrm{P} 2 \mathrm{VP}-1$, (c) $\mathrm{P}\left(\mathrm{St}-\mathrm{Fl}_{2}\right)-b$-P2 VP-2, (d) $\mathrm{P}\left(\mathrm{St}_{-} \mathrm{Fl}_{2}\right)-b-\mathrm{P} 2 \mathrm{VP}-3$. (e) TEM image of $\mathrm{P}\left(\mathrm{St}-\mathrm{Fl}_{2}\right)-b$-P2 VP-1 annealing at $280{ }^{\circ} \mathrm{C}$ for $24 \mathrm{~h}$.

e.g. $>280{ }^{\circ} \mathrm{C}$. The block copolymer series with longer polyfluorene units, $\mathrm{P}\left(\mathrm{St}-\mathrm{Fl}_{2}\right)-b$-P2VP, were also studied. Again, and rather unexpectedly, no X-ray peak was observed in the wide angle region (not shown), suggesting that also two fluorene units are not long enough to provide the block copolymers with liquid crystallinity on the $\mathrm{P}\left(\mathrm{St}-\mathrm{Fl}_{2}\right)$ block. Possibly, due to the alkyl chains emanating from the center of the fluorene unit, the aspect ratio of a double fluorene units mesogen is still not large enough to induce liquid crystallinity. The presence of two pendant fluorene units per PS monomer is, however, expected to greatly enhance the Flory-Huggins parameter, $\chi$, as well as increasing the stretching of PS backbone, thus affecting considerably the phase diagram.

Figure 3 shows the SAXS and TEM results for the P(St$\mathrm{Fl}_{2}$ )- $b$-P2VP block copolymers cast from toluene solutions and annealed at $230^{\circ} \mathrm{C}$. The SAXS peaks spaced as $1: 3^{1 / 2}: 2$ of $\mathrm{P}\left(\mathrm{St}-\mathrm{Fl}_{2}\right)-b-\mathrm{P} 2 \mathrm{VP}-1$ can be identified as a cylindrical structure with a lattice dimension of $25 \mathrm{~nm}$, consistent with the TEM image of Figure $3 \mathrm{~b} . \mathrm{P}\left(\mathrm{St}_{-} \mathrm{Fl}_{2}\right)-b-\mathrm{P} 2 \mathrm{VP}-2$ shows a poorly organized spherical structure in the corresponding TEM image, in agreement with the single broad peak observed in the SAXS. The SAXS and TEM results of $\mathrm{P}\left(\mathrm{St}-\mathrm{Fl}_{2}\right)-b-\mathrm{P} 2 \mathrm{VP}-3$ are both consistent with a lamellar structure with periodicity of $20 \mathrm{~nm}$. Interestingly, the TEM image in Figure $3 e$ reveals a gyroid phase when $\mathrm{P}\left(\mathrm{St}-\mathrm{Fl}_{2}\right)-b$ P2VP-1 is annealed at $280{ }^{\circ} \mathrm{C}$. This result may appear at first surprising since for fixed block copolymers volume fractions, mean-field theories do not predict gyroid phases at lower segregations than the cylindrical phase, that is, at higher temperatures. ${ }^{2}$ However, it is now well recognized both theoretically $^{19,20}$ and experimentally ${ }^{21,22}$ that fluctuations can stabilize gyroidal phases at lower segregation regimes than cylindrical phases. In the present case, the gyroidal phase has survived the cooling process from $280{ }^{\circ} \mathrm{C}$ to room temperature (at which TEM was performed) without transforming into the cylindrical phase observed at lower intermediate temperatures $\left(230^{\circ} \mathrm{C}\right.$ or lower $)$, due to the 
extremely slow kinetics of transformation of Ia3d gyroidal phases $\rightarrow$ cylindrical phases. This slow kinetics has already been extensively studied in previous literature. ${ }^{23} \mathrm{We}$ further note that the gyroid phase in symmetric block copolymers is extremely rare: we are only aware of one single example in the literature. $^{24}$

Figure 4 summarizes and sketches the phase behavior of $\mathrm{P}\left(\mathrm{St}-\mathrm{F} l_{n}\right)-b$-P2VP. As can be noted, at large volume fraction of $\mathrm{P}\left(\mathrm{St}-\mathrm{Fl} l_{n}\right)$, both the series $\mathrm{P}(\mathrm{St}-\mathrm{Fl})-b-\mathrm{P} 2 \mathrm{VP}$ and $\mathrm{P}\left(\mathrm{St}_{-} \mathrm{Fl}_{2}\right)-$ $b$-P2VP show spherical structures (S) with $\mathrm{P} 2 \mathrm{VP}$ forming the spheres. By moving toward more symmetric composition,

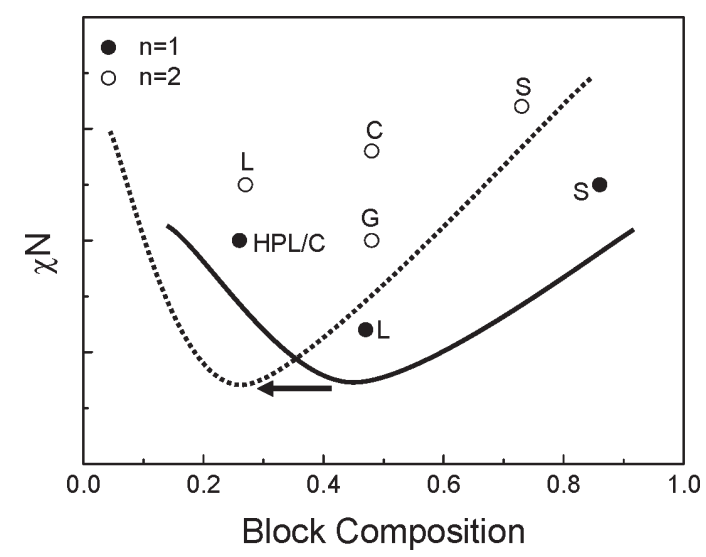

Figure 4. Effect of the side chain length on phase behavior of $\mathrm{P}\left(\mathrm{St}-\mathrm{Fl}_{n}\right)$ $b$-P2VP $(n=1,2)$. Solid line $(n=1)$ and dotted line $(n=2)$ are a guide for the eyes. however, the phase diagram changes. $\mathrm{P}(\mathrm{St}-\mathrm{Fl})-b$-P2VP with $f_{\mathrm{P}\left(\mathrm{St}-\mathrm{Fl}_{n}\right)}=0.47$ shows a lamellar (L) structure, while P(St$\left.\mathrm{Fl}_{2}\right)-b$-P2 VP with $f_{\mathrm{P}\left(\mathrm{St}-\mathrm{Fl}_{n}\right)}=0.48$ shows a cylindrical structure (C) at high $\chi N$ and a gyroid phase (G) at low $\chi N$. By further increasing the volume fraction of $\mathrm{P} 2 \mathrm{VP}, \mathrm{P}\left(\mathrm{St}_{-}-\mathrm{Fl}_{2}\right)-b-\mathrm{P} 2 \mathrm{VP}$ shows a lamellar structure at $f_{\mathrm{P}\left(\mathrm{St}_{-} \mathrm{Fl}_{2}\right)}=0.27$, while for identical composition, at $f_{\mathrm{P}(\mathrm{St}-\mathrm{Fl})}=0.27, \mathrm{P}(\mathrm{St}-\mathrm{Fl})-b-\mathrm{P} 2 \mathrm{VP}$ shows HPL or cylinders, depending on the initial solvent from which the sample has been cast. In other words, by increasing the fluorene side chain length, the phase diagram becomes highly asymmetric. This behavior can be understood by considering the conformational asymmetry of block copolymers, ${ }^{25}$ which can be represented by $\epsilon=$ $\left(\rho_{0 \mathrm{~A}} b_{\mathrm{A}} / \rho_{0 \mathrm{~B}} b_{\mathrm{B}}\right)^{2}$, where $\rho_{0}$ is the density and $b$ is the Kuhn statistical length of pure individual components. By attaching different lengths of fluorene chains to the PS block, the $\mathrm{P}$ $\left(\mathrm{St}-\mathrm{Fl}_{n}\right)$ block will experience an effective stretching and, thus, an increased persistence and Kuhn statistical length. Thus, it has to be expected that $\epsilon$ should vary as $\mathrm{P}\left(\mathrm{St}-\mathrm{Fl}_{2}\right)$ $b$-P2VP $>\mathrm{P}(\mathrm{St}-\mathrm{F} 1)-b$-P2VP $>\mathrm{PS}-b$-P2VP, leading to an increasingly asymmetric block copolymer with increasing length of Fl. Compared to the reference PS-P2VP system, the presence of a gyroid phase at nearly symmetric compositions for $\mathrm{P}\left(\mathrm{St}-\mathrm{Fl}_{2}\right)-b$-P2VP and that of HPL at very high $\mathrm{P} 2 \mathrm{VP}$ content in $\mathrm{P}(\mathrm{St}-\mathrm{Fl})-b-\mathrm{P} 2 \mathrm{VP}$ can be explained by the conformational asymmetry of the block copolymer. We further note that a highly asymmetric phase diagram is indeed expected for block copolymers in which one block has a very large persistence length ${ }^{26}$ and that when the persistence length is nearly "infinite", this leads to the self-assembly of rod-coil block copolymers in which liquid
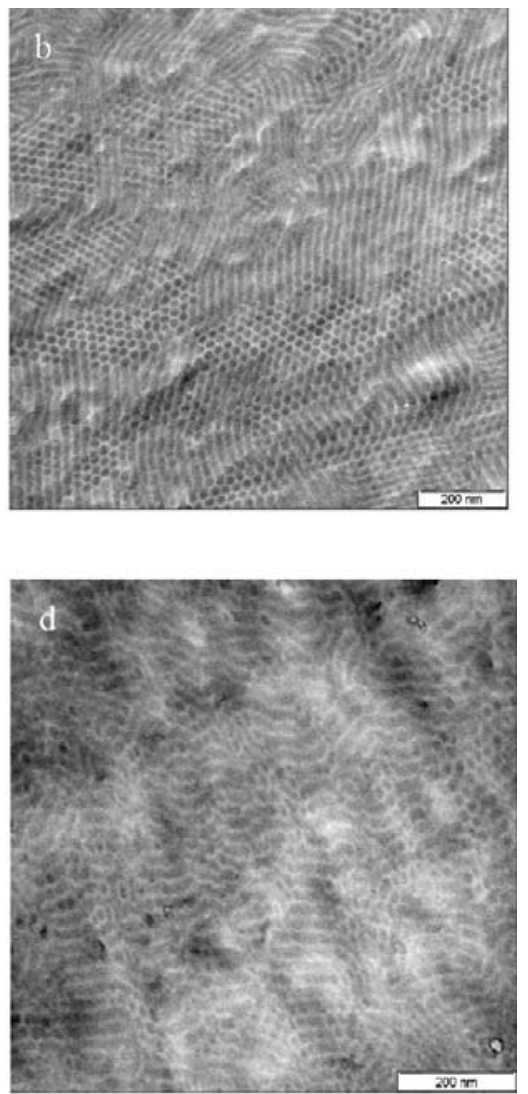

Figure 5. TEM images of $\mathrm{P}\left(\mathrm{St}_{-} \mathrm{Fl}_{2}\right)-b-\mathrm{P} 2 \mathrm{VP}-1$ and $\mathrm{P}\left(\mathrm{St}_{-}-\mathrm{Fl}_{2}\right)-b$-P2VP-1/P3-SWCNT $3.3 \mathrm{wt} \%$ as-cast and after thermal annealing at $170{ }^{\circ} \mathrm{C}$ for $24 \mathrm{~h}$ under high vacuum. (a) $\mathrm{P}\left(\mathrm{St}_{-}-\mathrm{Fl}_{2}\right)-b-\mathrm{P} 2 \mathrm{VP}-1$, as-cast from DMF, showing a lamellar phase. (b) $\mathrm{P}\left(\mathrm{St}-\mathrm{Fl}_{2}\right)-b-\mathrm{P} 2 \mathrm{VP}-1$, after thermal annealing showing a cylindrical phase. (c) P(St-Fl 2$)-b$-P2VP-1/P3-SWCNT $3.3 \mathrm{wt} \%$, as-cast from DMF. (d) P(St-Fl 2$)-b$-P2VP-1/P3-SWCNT 3.3 wt \%, after thermal annealing. 

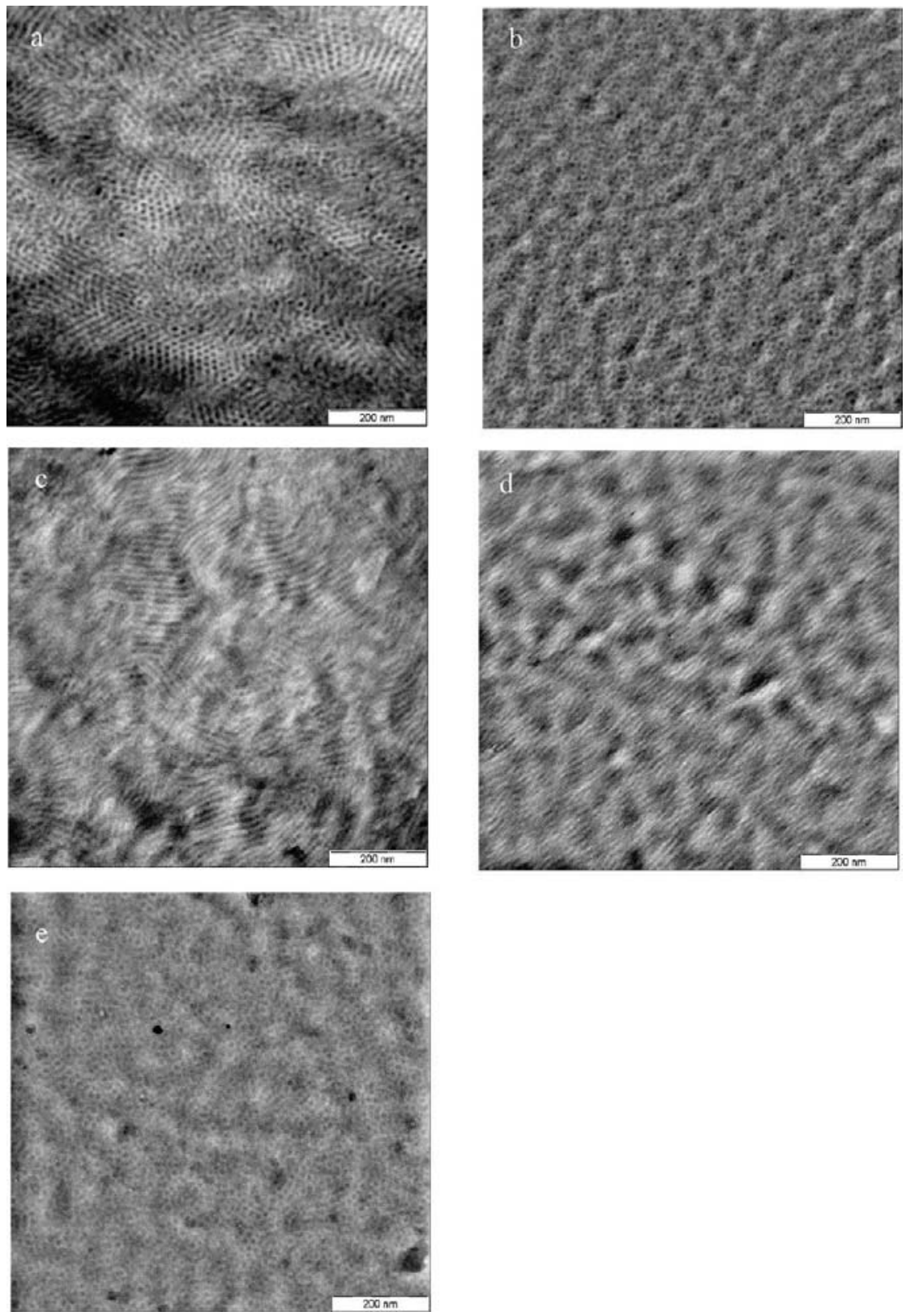

Figure 6. TEM images of $\mathrm{P}\left(\mathrm{St}-\mathrm{Fl}_{2}\right)-b-\mathrm{P} 2 \mathrm{VP}-2$ and $\mathrm{P}\left(\mathrm{St}_{-}-\mathrm{Fl}_{2}\right)-b$-P2 $\mathrm{VP}-2 / \mathrm{P} 3-\mathrm{SWCNT} 1.0 \mathrm{wt} \%$ as-cast from $\mathrm{DMF}$ and after further thermal annealing at $170{ }^{\circ} \mathrm{C}$ for $24 \mathrm{~h}$ under high vacuum. (a) $\mathrm{P}\left(\mathrm{St}-\mathrm{Fl}_{2}\right)-b-\mathrm{P} 2 \mathrm{VP}-2$, as cast. (b) $\mathrm{P}\left(\mathrm{St}-\mathrm{Fl} \mathrm{l}_{2}\right)-b$-P2VP-2, after annealing at $170{ }^{\circ} \mathrm{C}$ for $24 \mathrm{~h}$. (c) $\mathrm{P}\left(\mathrm{St}-\mathrm{Fl} l_{2}\right)-b$ - $\mathrm{P} 2 \mathrm{VP}-2 /$ P3-SWCNT $1.0 \mathrm{wt} \%$, as casted. (d) P(St-Fl $)$-b-P2VP-2/P3-SWCNT $1.0 \mathrm{wt} \%$, after annealing at $170{ }^{\circ} \mathrm{C}, 24 \mathrm{~h}$. (e) $\mathrm{P}(\mathrm{St}-\mathrm{Fl} 2)-b-\mathrm{P} 2 \mathrm{VP}-2 / \mathrm{P} 3-\mathrm{SWCNT}$ $1.0 \mathrm{wt} \%$, after annealing at $230{ }^{\circ} \mathrm{C}$ for $24 \mathrm{~h}$.

crystalline interactions also contribute to the final phase diagram. ${ }^{27}$

3.3. Effect of Carbon Nanotubes on Phase Behavior of $\mathbf{P}\left(\mathbf{S t}_{-\mathbf{F l}}\right)-\boldsymbol{b}$-P2VP. Because of extended van der Waals and $\pi-\pi$ interactions, CNTs are well-known to have a strong tendency to aggregate into insoluble bundles. In order to overcome such solubility problems, covalent modification or adsorption of soluble molecules on CNTs has been attempted as a solubilization technique. In what follows carboxylic-modified SWCNTs are used instead of pristine SWCNTs. The use of $\mathrm{COOH}$-modified carbon nanotubes offers a twofold advantage: first, an improved dispersibility in DMF is expected, ${ }^{28}$ and second, carboxylic acid groups should in principle interact with the pyridine moiety in $\mathrm{P}\left(\mathrm{St}-\mathrm{Fl}{ }_{n}\right)-b$-P2VP. ${ }^{29}$ In addition, polyvinylpyridines have been already shown to offer good hosting capabilities for electron acceptor moieties ${ }^{30}$ and electron conductive polymers. ${ }^{31}$ While doing this, it should be kept in mind, however, that DMF is a selective solvent for $\mathrm{P}\left(\mathrm{St}-\mathrm{Fl} l_{n}\right)-b-\mathrm{P} 2 \mathrm{VP}$, which preferentially swells the $\mathrm{P} 2 \mathrm{VP}$ domains.

Figures $5 \mathrm{a}$ and $6 \mathrm{a}$ show respectively the morphologies of $\mathrm{P}\left(\mathrm{St}-\mathrm{Fl}_{2}\right)-b$-P2VP-1 and $\mathrm{P}\left(\mathrm{St}_{-}-\mathrm{Fl}_{2}\right)-b-\mathrm{P} 2 \mathrm{VP}-2$ after being cast from the DMF solutions and being dried at $70{ }^{\circ} \mathrm{C}$ (lower than the glass transitions of both the blocks) under high vacuum. Figures $5 \mathrm{~b}$ and $6 \mathrm{~b}$ show respectively the same samples after further thermal annealing at $170{ }^{\circ} \mathrm{C}$ for $24 \mathrm{~h}$. After casting, $\mathrm{P}\left(\mathrm{St}_{-} \mathrm{Fl}_{2}\right)-b$-P2VP-1 shows a lamellar structure (Figure 5a) while $\mathrm{P}\left(\mathrm{St}-\mathrm{Fl}_{2}\right)-b-\mathrm{P} 2 \mathrm{VP}-2$ shows a cylindrical structure (Figure 6a). After annealing at $170{ }^{\circ} \mathrm{C}$, both the block copolymers undergo an order-order transition (OOT): the lamellar phase evolves into a cylindrical structure for $\mathrm{P}\left(\mathrm{St}_{-}-\mathrm{Fl}_{2}\right)-b$ P2VP-1 (Figure 5b), while the cylindrical structure evolves to spherical morphology for $\mathrm{P}\left(\mathrm{St}_{-} \mathrm{Fl}_{2}\right)-b-\mathrm{P} 2 \mathrm{VP}-2$ (Figure 6b). 
(a)

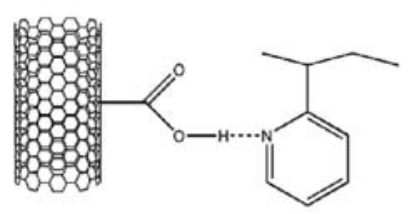

(b)

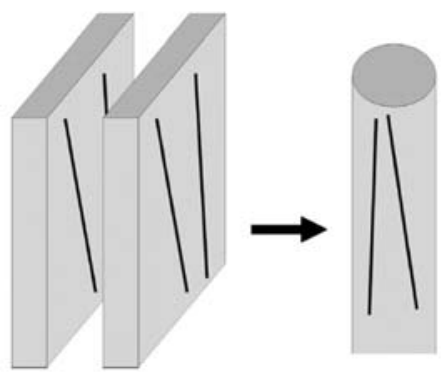

$\mathbf{L}$

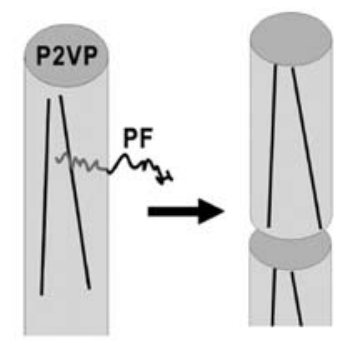

Intermediate state

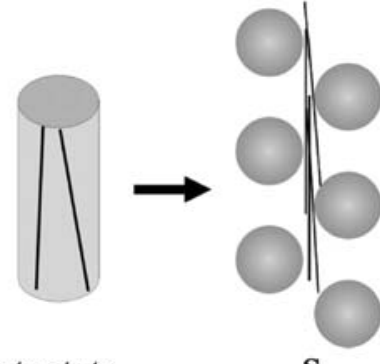

$\mathbf{S}$

Figure 7. Schematic drawing of (a) the interactions between carbon nanotubes and P2VP and (b) effect of carbon nanotube on the OOTs of $\mathrm{P}\left(\mathrm{St}-\mathrm{Fl}{ }_{n}\right)-b-\mathrm{P} 2 \mathrm{VP}$.

Figures $5 \mathrm{c}$ and $6 \mathrm{c}$ show respectively the morphologies of $\mathrm{P}\left(\mathrm{St}-\mathrm{Fl}_{2}\right)-b$-P2VP-1 and $\mathrm{P}\left(\mathrm{St}-\mathrm{Fl}_{2}\right)-b$ - $\mathrm{P} 2 \mathrm{VP}-2$ blended with a very low amount of $\mathrm{COOH}-\mathrm{SWCNT}$ after casting from DMF and drying at $70{ }^{\circ} \mathrm{C}$ under high vacuum. Figures $5 \mathrm{~d}$ and $6 \mathrm{~d}$ show respectively the same blends after successive thermal annealing at $170{ }^{\circ} \mathrm{C}$ for $24 \mathrm{~h}$. The clear dots in the TEM images can be attributed to the partial segregation of CNTs into distinct domains, inferring that part of CNTs phase segregated in the block copolymers. When compared with Figures $5 \mathrm{a}, \mathrm{b}$ and $6 \mathrm{a}, \mathrm{b}$, it can be clearly appreciated that a very low amount of SWCNTs is sufficient to induce poor ordering in the final morphologies. Since the length of carbon nanotubes $\left(\sim 10^{3} \mathrm{~nm}\right.$ length) is much larger than the periodicities of the block copolymers considered in the present study $\left(\sim 10^{1} \mathrm{~nm}\right)$, poorly organized structures are expected to arise as a direct consequence of the perturbations of carbon nanotubes on the structure organizing process of the block copolymers.

Particularly enlightening is the transition from Figure $6 c$ to Figure 6d. Differently from the unloaded $\mathrm{P}\left(\mathrm{St}_{-}-\mathrm{Fl}_{2}\right)-b$ P2VP-2 (Figure 6a,b), when COOH-SWCNTs are added, the transition cylinders $\rightarrow$ spheres does not take place upon annealing at $170{ }^{\circ} \mathrm{C}$, with the blend remaining in some form of intermediate cylindrical structure, where the cylinders are broken into portions with shorter lengths. Only upon further annealing at $230^{\circ} \mathrm{C}$ for $24 \mathrm{~h}$ under ultrahigh vacuum (ca. $10^{-8}$ $\mathrm{mbar}$ ), the intermediate cylindrical structure transforms into the expected spherical structure.

This phenomenon can be understood considering the interactions between CNTs and P2VP. The carboxylic groups on the CNT walls induce, via selective hydrogen bonds (see Figure 7a), preferential partitioning of CNTs in the P2VP domains. Furthermore, because of linear, single dimensionality of CNTs $\left(\sim 10^{3} \mathrm{~nm}\right.$ length), these can be dispersed in one- and two-dimensional phases, such as the lamellar and cylindrical phases. However, dispersion in zerodimensional phases such as spheres is expected to be difficult, as the usual space periodicity of the block copolymers $\left(\sim 10^{2} \mathrm{~nm}\right)$ is much smaller than the CNT lengths. Therefore, by annealing at $170{ }^{\circ} \mathrm{C}, \mathrm{P}\left(\mathrm{St}_{-}-\mathrm{Fl}_{2}\right)-b-\mathrm{P} 2 \mathrm{VP}-1$ could easily rearrange from lamellar to cylinders (Figure $5 \mathrm{c}, \mathrm{d}$ ). On the

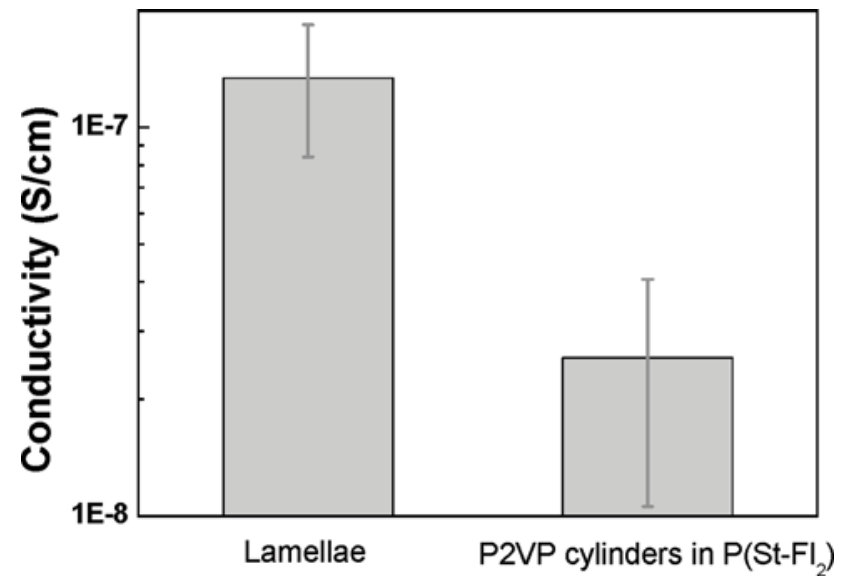

Figure 8. Effect of morphology on the conductivity of $\mathrm{P}\left(\mathrm{St}-\mathrm{Fl}_{n}\right)-b$ $\mathrm{P} 2 \mathrm{VP} / \mathrm{P} 3-\mathrm{SWCNT}(2$ wt \%). P(St-Fl)-b-P2VP-1 shows the lamellae structure, and $\mathrm{P}\left(\mathrm{St}_{-} \mathrm{Fl}_{2}\right)-b-\mathrm{P} 2 \mathrm{VP}-1$ shows the $\mathrm{P} 2 \mathrm{VP}$ cylinders in the $\mathrm{P}\left(\mathrm{St}-\mathrm{Fl}_{2}\right)$ matrix.

contrary, $\mathrm{P}\left(\mathrm{St}_{-}-\mathrm{Fl}_{2}\right)-b-\mathrm{P} 2 \mathrm{VP}-2$ could not easily evolve into a spherical structure, leading to an intermediate ill-defined cylindrical structure (Figure 6d). Only upon further annealing at $230{ }^{\circ} \mathrm{C}$, where the hydrogen-bonding interactions between CNTs and P2VP are completely suppressed, CNTs were found to segregate and $\mathrm{P}\left(\mathrm{St}-\mathrm{Fl}_{2}\right)-b-\mathrm{P} 2 \mathrm{VP}-2$ could evolve into the final spherical structure. Figure $7 \mathrm{~b}$ schematically sketches these findings.

In order to further assess how well the SWCNTs were dispersed within the P2VP domains, we measured the conductivity of $\mathrm{P}(\mathrm{St}-\mathrm{Fl})-b-\mathrm{P} 2 \mathrm{VP}-1$ and $\mathrm{P}\left(\mathrm{St}_{-} \mathrm{Fl}_{2}\right)-b-\mathrm{P} 2 \mathrm{VP}-1$ blending with $2 \mathrm{wt} \% \mathrm{COOH}-\mathrm{SWCNT}$. Here both the block copolymers have the same P2VP composition $(0.52-0.53)$, while $\mathrm{P}(\mathrm{St}-\mathrm{Fl})-b-\mathrm{P} 2 \mathrm{VP}-1$ showed the lamellar structure and $\mathrm{P}\left(\mathrm{St}_{-} \mathrm{Fl}_{2}\right)-b-\mathrm{P} 2 \mathrm{VP}-1$ showed the cylindrical structure. As shown in Figure 8, addition of nanotubes to the block copolymers increased the ac conductivity of the samples from $\approx 0\left(10^{-9} \mathrm{~S} / \mathrm{cm}\right.$ is the thereshold of the instrument $)$ up to $10^{-7} \mathrm{~S} / \mathrm{cm}$. This supports partial dispersion of CNTs; nonetheless, this conductivity is still low compared 
to polymer composites with similar CNT concentration $\left(10^{-4} \mathrm{~S} / \mathrm{cm}\right) .^{32}$ While this can be related to a residual aggregation of SWCNT within the P2VP domains, the highly anisotropy of the block copolymer system, when compared with polyethylene-SWCNT composites reported in ref 32 , can also play a role. In this respect Figure 8 shows a progressive depression of the conductivity from $10^{-7}$ to $10^{-8}$ $\mathrm{S} / \mathrm{cm}$ when going from lamellar $\rightarrow$ cylinder (P2VP forms the cylinders in $\mathrm{P}\left(\mathrm{St}_{-} \mathrm{Fl}_{2}\right)-b$ - $\left.\mathrm{P} 2 \mathrm{VP}-1\right)$, consistent with twodimensional versus one-dimensional conductivity mechanisms. A similar topological dependence on the structure of the block copolymers has been already demonstrated and discussed in proton-conductive systems. ${ }^{33}$

\section{Conclusions}

We have presented the synthesis and self-assembly behavior of a new type of block copolymer, $\mathrm{P}\left(\mathrm{St}-\mathrm{Fl}_{n}\right)-b$-P2 VP, in which one block is a coiled P2VP, while the other block is a stretched, semiflexible PS chain bearing, for each monomer, a mesogen of one or two fluorene units. The main effect of the fluorene units is twofold: (i) they increase the phase segregation parameter, $\chi N$, and (ii) they increase the Khun length of the PS block. When compared with the reference PS-P2VP block copolymer system, the phase behavior of $\mathrm{P}\left(\mathrm{St}-\mathrm{F} l_{n}\right)-b$ - $\mathrm{P} 2 \mathrm{VP}$ can be understood in terms of the conformational asymmetry of block copolymers arising from the grafting of fluorene units onto the backbone of the PS block. The final phase diagram for these block copolymers shows spherical structures, hexagonally packed cylinders, lamellar, gyroidal, and hexagonally perforated lamellae, depending on the molecular conformation of the block copolymers.

We have further investigated the possibility to use the present block copolymers to disperse single wall carbon nanotubes (SWCNTs). In order to best exploit supramolecular binding interactions, COOH-modified SWCNTs were used. The hydrogen bonding between $\mathrm{COOH}$ and P2VP moieties effectively allows for an improved dispersibility of the SWCNT within the P2VP domains, as revealed by systematic shifts in the orderorder transitions when the block copolymers are blended with the SWCNTs. The capability to encapsulate SWCNT was also found to be directly dependent on the microphase-separated structure of the block copolymers: two-dimensional (lamellae) structure being more efficient than one-dimensional morphology (cylinders) in this task. Electron conductivity measurements confirmed the morphology-dependent efficiency of the block copolymers in the encapsulation of SWCNTs, although relatively low conductivity values suggested residual aggregation within the P2VP.

In summary, this study presents a possible new system to access a broad range of block copolymer morphologies and allows for facile tuning of the electrical properties by blending with suitably functionalized carbon nanotubes.

Supporting Information Available: Experimental details.

\section{References and Notes}

(1) Bower, D. I. An Introduction to Polymer Physics; Cambridge University Press: New York, 2002.

(2) Leibler, L. Macromolecules 1980, 13, 1602

(3) (a) Matsen, M. W.; Bates, F. S. J. Polym. Sci., Part B: Polym. Phys. 1997, 35, 945. (b) Bockstaller, M. R.; Mickiewicz, R. A.; Thomas, E. L. Adv. Mater. 2005, 17, 1331 .

(4) (a) McCrackin, F. L.; Mazur, J. Macromolecules 1981, 14, 1214. (b) Rouault, Y.; Borisov, O. V. Macromolecules 1996, 29, 2605.
(5) Hammond, M. R.; Mezzenga, R. Soft Matter 2008, 4, 952.

(6) (a) Dudowicz, J.; Freed, K. F. Macromolecules 2000, 33, 52925299. (b) Ruzette, A. G.; Banerjee, P.; Mayes, A. M.; Pollard, M.; Russell, T. P.; Jerome, R.; Slawecki, T.; Hjelm, R.; Thiyagarajan, P. Macromolecules 1998, 31, 8509.

(7) (a) Ruokolainen, J.; Brinke, G. T.; Ikkala, O. Adv. Mater. 1999, 11, 777. (b) Ruokolainen, J.; Mäkinen, R.; Torkkeli, M.; Mäkelä, T.; Serimaa, R.; Brinke, G.; Ikkala, O. Science 1998, 280, 557.

(8) (a) Canilho, N.; Kasemi, E.; Mezzenga, R.; Schluter, A. D. J. Am. Chem. Soc. 2006, 128, 13998. (b) Canilho, N.; Kasemi, E.; Schluter, A. D.; Mezzenga, R. Macromolecules 2007, 40, 2822. (c) Canilho, N.; Scholl, M.; Klok, H.; Mezzenga, R. Macromolecules 2007, 40, 8374. (d) Mezzenga, R.; Ruokolainen, J.; Canilho, N.; Kasemi, E.; Schluter, D. A.; Lee, W. B.; Fredrickson, G. H. Soft Matter 2009, 5, 92.

(9) Li, C.; Schluter, A. D.; Zhang, A.; Mezzenga, R. Adv. Mater. 2008, 20,4530 .

(10) (a) Wu, P.; Ren, G.; Li, C.; Mezzenga, R.; Jenekhe, S. A. Macromolecules 2009, 42, 2317. (b) Rubatat, L.; Kong, X.; Jenekhe, S. A.; Ruokolainen, J.; Hojeij, M.; Mezzenga, R. Macromolecules 2008, 41, 1846. (c) Dai, C.; Yen, W.; Lee, Y.; Ho, C.; Su, W. J. Am. Chem. Soc. 2007, 129, 11036. (d) Surin, M.; Marsitzky, D.; Grimsdale, A.; Müllen, K.; Lazzaroni, R.; Leclère, P. Adv. Funct. Mater. 2004, 14, 708. (e) Lu, S.; Liu, T.; Ke, L.; Ma, D.; Chua, S.; Huang, W. Macromolecules 2005, 38,8494 .

(11) (a) Jung, Y. C.; Sahoo, N. G.; Cho, J. W. Macromol. Rapid Commun. 2006, 27, 126. (b) Zou, J.; Khondaker, S. I.; Huo, Q.; Zhai, L. Adv. Funct. Mater. 2009, 19, 479.

(12) Kong, H.; Gao, C.; Yan, D. J. Am. Chem. Soc. 2004, 126, 412.

(13) Kanibolotsky, A. L.; Berridge, R.; Skabara, P. J.; Perepichka, I. F.; Bradley, D. D. C.; Koeberg, M. J. Am. Chem. Soc. 2004, 126, 13695.

(14) Sugiyama, K.; Hirao, A.; Hsu, J. C.; Tung, Y. C.; Chen, W. C. Macromolecules. 2009, 42, 4053.

(15) Hajduk, D. A.; Takenouchi, H.; Hillmyer, M. A.; Bates, F. S.; Vigild, M. E.; Almdal, K. Macromolecules 1997, 30, 378.

(16) (a) Matsen, M. W.; Bates, F. S. Macromolecules 1996, 29, 1091. (b) Matsen, M. W.; Bates, F. S. Macromolecules 1996, 29, 7641.

(17) Schulz, M. F.; Khandpur, A. K.; Bates, F. S.; Almdal, K.; Mortensen, K.; Hajduk, D. A.; Gruner, S. M. Macromolecules 1996, 29, 2857.

(18) Zha, W.; Han, C. D.; Lee, D. H.; Han, S. H.; Kim, J. K.; Kang, J. H.; Park, C. Macromolecules 2007, 40, 2109.

(19) Podneks, V. E.; Hamley, I. W. JETP Lett. 1996, 271, 976

(20) Hamley, I. W.; Podneks, V. E. Macromolecules 1997, 30, 3701.

(21) Vigild, M. E.; Almdal, K.; Mortensen, K.; Hamley, I. W.; Fairclough, J. P. A.; Ryan, A. J. Macromolecules 1998, 31, 5702.

(22) Bodycomb, J.; Yamaguchi, D.; Hashimoto, T. Macromolecules 2000, 35, 5187 .

(23) (a) Bodycomb, J.; Yamaguchi, D.; Hashimoto, T. Macromolecules 2000, 33, 5187. (b) Laurer, J. H.; Hajduk, D. A.; Fung, J. C.; Sedat, J. W.; Smith, S. D.; Gruner, S. M.; Agard, D. A.; Spontak, R. J. Macromolecules 1997, 30, 3938. (c) Hajduk, D. A.; Harper, P. E.; Gruner, S. M.; Honeker, C. C.; Kim, G.; Thomas, E. L.; Fetters, L. J. Macromolecules 1994, 27, 4063. (d) Förster, S.; Khandpur, A. K.; Zhao, J.; Bates, F. S.; Hamley, I. M.; Ryan, A. J.; Bras, W. Macromolecules 1994, 27, 6922.

(24) Park, M. J.; Balsara, N. Macromolecules 2008, 41, 3678.

(25) (a) Bates, F. S.; Fredrickson, G. H. Macromolecules 1994, 27, 1065. (b) Bates, F. S.; Schulz, M. F.; Khandpur, A. K.; Forster, S.; Rosedale, J. H.; Almdal, K.; Mortensen, K. Faraday Discuss. 1994, 98, 7.

(26) Reenders, M.; ten Brinke, G. Macromolecules 2002, 35, 3266.

(27) (a) Pryamitsyn, V.; Ganesan, V. J. Chem. Phys. 2004, 120, 5824. (b) Sary, N.; Rubatat, L.; Brochon, C.; Hadziioannou, G.; Ruokolainen, J.; Mezzenga, R. Macromolecules 2007, 40, 6990.

(28) Zhao, B.; Hu, H.; Yu, A.; Perea, D.; Haddon, R. C. J. Am. Chem. Soc. 2005, 127, 8197.

(29) Fujita, N.; Yamashita, T.; Asai, M.; Shinkai, S. Angew. Chem., Int. Ed. 2005, 44, 1257.

(30) Laiho, A.; Ras, R. H. A.; Valkama, S.; Ruokolainen, J.; Osterbacka, R.; Ikkala, O. Macromolecules 2006, 39, 7648.

(31) Mezzenga, R.; Ruokolainen, J.; Fredrickson, G. H.; Kramer, E. J.; Moses, D.; Heeger, A. J.; Ikkala, O. Science 2003, 299, 1872.

(32) Haggenmueller, R.; Guthy, C.; Lukes, J. R.; Fischer, J. E.; Winey, K. I. Macromolecules 2007, 40, 2417.

(33) Rubatat, L.; Li, C.; Dietsch, H.; Nykänen, A.; Ruokolainen, J.; Mezzenga, R. Macromolecules 2008, 41, 8130. 